\title{
Using nitrogen-vacancy defects in diamond as in-situ sensors of band bending.
}

\author{
Spatial mapping of band bending in semiconductor devices using in situ quantum \\ sensors. Nat. Electron. 1, 502-507 (2018). \\ Authors: D.A. Broadway, N. Dontschuk, A. Tsai, S. E. Lillie, C. T.-K. Lew, J. C. \\ McCallum, B. C. Johnson, M. W. Doherty, A. Stacey, L. C. L. Hollenberg \& J.-P. Tetienne. \\ Nat. Electron. 1, 502-507 (2018).
}

\section{Recommended with a Commentary by Toeno van der Sar, Kavli Institute of Nanoscience, Delft University of Technology}

When one material is connected to another, charges near the interface can reorganize to lower the energy of the system. The resulting space-dependent electrostatic potential near the interface alters the local electronic band structure, a phenomenon known as band bending. Band bending is an important concept for understanding the charge transport properties of material interfaces and nanoelectronic devices. It is central to the working of PN diodes, determines our ability to create Ohmic contacts between metals and semiconductors, and causes electronic properties of surfaces to differ from those in the material's bulk.

The degree of band bending at material surfaces can be hard to predict because of the potential presence of uncontrolled electronic states near the surface. In addition, band-bended regions typically have a nanometer-scale spatial extent, rendering them technically challenging to characterize. Broadway et al. introduce a technique to directly measure the electric field associated with band bending in-situ ${ }^{1}$. Their experimental concept is to use atomic-scale lattice defects inside the band-bended region as electrometers to directly quantify the local electric fields.

To do so, the authors created nitrogen-vacancy (NV) lattice defects in diamond inside the band-bended region near a diamond-air interface. NV defects have energy states in the diamond band gap and can exist in various charge states depending on the location of the Fermi energy. The negatively charged NV state is famous for its $\mathrm{S}=1$ electronic spin that can be initialized and read out optically and manipulated with high fidelity via electron spin resonance techniques ${ }^{2}$. The spin of the NV- defect is now used around the world as a nanoscale sensor of magnetic fields, with emerging applications to condensed-matter physics ${ }^{3}$. At the same time, the NV spin transition frequencies are also sensitive to electric fields ${ }^{4}$, making them excellent candidates to probe a phenomenon like band-bending in-situ. Compared to NV magnetometry, NV-electrometry is a relatively less-explored technique that is now starting to realize some of its opportunities for probing condensed-matter systems on the nanoscale. Probing band-bending as is done in this work is a beautiful example of a new and paradigmatic condensed-matter physics application of the NV sensor.

The authors created a dense layer of NV centers at $\sim 10 \mathrm{~nm}$ below the diamond surface and quantified the local electric field through measurements of the ESR spectrum of these NV centers. They explicitly show that the electric field associated with band bending gets modified by procedures such as acid-cleaning and 
oxygen-plasma-etching. This is important for applications that aim at using shallow NV centers as magnetic sensors, as excessive electric fields cause the $\mathrm{NV}^{-}$center to lose its electron and thereby the optical addressability of its spin state. From the measured electric fields they estimated charge densities at the diamond surface of $\sim 0.1 / \mathrm{nm}^{2}$, consistent with recent work by the authors using X-ray absorption spectroscopy ${ }^{5}$.

In addition to quantifying band-bending near the diamond-air surface, the authors also measured the electric fields while forcing an electric current through the diamond with electrodes on the surface. In particular, they spatially mapped the in-diamond electric field parallel to the diamond surface associated with this current. The results are very surprising, showing an abrupt decrease in electric field at some distance from the injection electrode. Without going into the complex underlying mechanisms here, I find these measurements intriguing because they demonstrate that the power of spatial imaging by revealing unexpected spatial dependencies of the electric fields associated with charge transport in a sample.

Finally, the supplementary material analyzes the procedure and challenges of using NV centers to characterize near-surface electric fields in detail. It considers for instance the role of strain, which can affect the ESR transitions similar to electric fields. Furthermore, it comments on various possible surface terminations of the diamond and describes the associated details of the near-interface band bending and charge stability of the NV centers.

An important question that remains is if the approach can be applied to other materials. After all, it does require suitable quantum sensors to exist in a material of interest. Candidate materials with suitable defects include silicon and silicon carbide. Fortunately, semiconductors host an enormous variety of defects, providing hope for finding a defect with similarly powerful in-situ sensing capabilities.

\section{References}

1. Broadway, D. A., Dontschuk, N., Tsai, A., Lillie, S. E., Lew, C. T.-K., McCallum, J. C., Johnson, B. C., Doherty, M. W., Stacey, A., Hollenberg, L. C. L. \& Tetienne, J.-P. Spatial mapping of band bending in semiconductor devices using in situ quantum sensors. Nat. Electron. 1, 502-507 (2018).

2. Rondin, L., Tetienne, J.-P., Hingant, T., Roch, J.-F., Maletinsky, P. \& Jacques, V. Magnetometry with nitrogen-vacancy defects in diamond. Reports Prog. Phys. 77, 056503 (2014).

3. Casola, F., van der Sar, T. \& Yacoby, A. Probing condensed matter physics with magnetometry based on nitrogen-vacancy centres in diamond. Nat. Rev. Mater. 3, 17088 (2018).

4. Dolde, F., Fedder, H., Doherty, M. W., Nöbauer, T., Rempp, F., Balasubramanian, G., Wolf, T., Reinhard, F., Hollenberg, L. C. L., Jelezko, F. \& Wrachtrup, J. Electric-field sensing using single diamond spins. Nat. Phys. 7, 459-463 (2011).

5. Stacey, A., Dontschuk, N., Chou, J.-P., Broadway, D. A., Schenk, A., Sear, M. J., Tetienne, J.-P., Hoffman, A., Prawer, S., Pakes, C. I., Tadich, A., de Leon, N. P., Gali, A. \& Hollenberg, L. C. L. Evidence for Primal sp2 Defects at the Diamond Surface: Candidates for Electron Trapping and Noise Sources. (2018). at <http://arxiv.org/abs/1807.02946> 